\title{
Efeito da modificação térmica nas propriedades físicas da madeira de Pinus Caribaea var. Hondurensis Barrett \& Golfari
}

\author{
Vaniele Bento dos Santos ${ }^{1 *}$; Gilmar Correia Silva ${ }^{2}$
}

DOI: https://doi.org/10.35699/2447-6218.2021.26590

\begin{abstract}
Resumo
A madeira do gênero Pinus é amplamente utilizada na indústria florestal, principalmente no setor moveleiro e civil, e por isso, faz-se necessário a busca por técnicas que melhorem e aperfeiçoem as suas propriedades tecnológicas. Neste sentido, o presente estudo teve como objetivo avaliar o efeito da modificação térmica nas propriedades físicas da madeira de Pinus caribaea var. hondurensis sob duas diferentes temperaturas. Foram utilizadas três árvores com idade de 14 anos, coletadas no campo experimental da Universidade Estadual do Sudoeste da Bahia, em Vitória da Conquista, BA, das quais foram produzidas amostras com dimensões de 30 x 30 x $50 \mathrm{~mm}$. O processo de modificação térmica foi realizado em uma estufa laboratorial a $160^{\circ} \mathrm{C}$ e $200^{\circ} \mathrm{C}$ de temperatura final de ciclo que, somados ao controle, representaram três tratamentos em análise. Foram realizados os ensaios de densidade aparente, umidade, perda de massa, taxa de absorção de água e estabilidade dimensional. Concluiu-se que a modificação térmica alterou as propriedades físicas avaliadas, tornando a madeira menos higroscópica e com maior estabilidade dimensional, além de ter influenciado na coloração da madeira em relação a sua aparência natural.
\end{abstract}

Palavras-chave: Estabilidade dimensional. Higroscopia. Termorretificação. Tratamento térmico.

\section{Effect of thermal modification on the physical properties of Pinus caribaea var. hondurensis Barrett \& Golfari wood}

\begin{abstract}
Pinus wood is widely used in the forestry industry, mainly in the furniture and civil sector, and for that reason, it is necessary to search for techniques that improve and perfect its technological properties. In this sense, the present study aimed to evaluate the effect of thermal modification on the physical properties of Pinus caribaea var. hondurensis under two different temperatures. Three trees were used at the age of 14, collected in the experimental field of the State University of Southwest Bahia, in Vitória da Conquista, BA, of which they were produced with dimensions of $30 \times 30 \times 50 \mathrm{~mm}$. The thermal modification process was carried out in a laboratory oven at $160^{\circ} \mathrm{C}$ and $200^{\circ} \mathrm{C}$ of final cycle temperature, which, added to the control, represented three treatments under analysis. The data of apparent density, humidity, loss of mass, water absorption rate and dimensional stability were performed. It was concluded that the thermal modification altered the physical properties evaluated, making the wood less hygroscopic and with greater dimensional stability, in addition to influencing the color of the wood in relation to its natural appearance.
\end{abstract}

Keywords: Dimensional stability. Heat treatment. Hygroscopy. Thermorectification.

${ }^{1}$ Universidade Federal de Minas Gerais. Instituto de Ciências Agrárias. Montes Claros, MG. Brasil. https://orcid.org/0000-0003-2391-1096

${ }^{2}$ Universidade Estadual do Sudoeste da Bahia. Vitória da Conquista, Ba. Brasil. https://orcid.org/0000-0002-8931-8773

*Autor correspondente: vanielebento@hotmail.com

Recebido para publicação em 07 de dezembro de 2020. Aceito para publicação em 19 de janeiro de 2021. e-ISSN: 2447-6218 / ISSN: 2447-6218. Atribuição CC BY. 


\section{Introdução}

A madeira é um material orgânico e heterogêneo que pode apresentar restrições de uso a depender do produto final devido a algumas propriedades intrínsecas, como higroscopicidade, anisotropia e susceptibilidade ao ataque biológico. A sua higroscopicidade está relacionada à estrutura química da parede celular, onde contém os polímeros de celulose, hemicelulose e lignina, que formam os grupos hidroxílicos. Dentre esses polímeros, a hemicelulose é a mais hidrófila favorecendo a troca de água com o meio e, consequentemente, a variação dimensional da madeira (Hill, 2006; Modes et al., 2017).

E dentre as tecnologias utilizadas para melhorar a qualidade e uso da madeira, encontra-se a modificação térmica, a qual consiste em um processamento da madeira a altas temperaturas com o propósito de alterar a sua composição química e proporcionar melhorias em suas propriedades, como por exemplo, a cor, estabilidade dimensional e a vantagem ambiental de aumentar a resistência à biodeterioração da madeira sem a impregnação ou utilização de produtos químicos (Batista, 2019).

O processo de modificação térmica, geralmente, é realizado com temperaturas de 150 a $280^{\circ} \mathrm{C}$ e períodos de tempo variando entre 15 minutos a 24 horas, a depender das características do material, do processo utilizado e do aspecto desejado ao produto final (Kamdem et al., 2002). Entretanto, dependendo da temperatura e tempo utilizado no processo, pode ocorrer a perda da resistência mecânica do material. De acordo Moura et al., (2011), madeiras de Pinus caribaea var. hondurensis quando submetidas a modificação térmica com temperaturas acima de $200^{\circ} \mathrm{C}$, são necessários cuidados adicionais em situações nas quais a resistência ao cisalhamento seja um fator importante.

Visto que o lenho do gênero Pinus tende a apresentar algumas limitações tecnológicas, como alto grau de higroscopicidade, a madeira deste gênero têm sido uma das mais utilizadas no processo de modificação térmica com o intuito de agregar maior valor ao produto final. Segundo a International ThermoWood Association (2020), $48 \%$ da madeira tratada pelo processo ThermoWood ${ }^{\circledR}$ no ano de 2019, foi representada pelas espécies de pinus. No continente europeu, a madeira submetida a este tratamento térmico tem uma larga aplicação para usos ao ar livre, como decks, móveis para jardim, componentes de portas e janelas, bem como, mobiliário interno, estruturas de saunas e banheiros (Esteves e Pereira, 2009).

Embora esse processo não esteja consolidado no Brasil, é evidente a importância do gênero de Pinus para o país, já que, dos 9 milhões de hectares de árvores plantadas no território nacional, 1,64 milhão de hectares são de espécies de pinus. No ano de 2019, os plantios de pinus apresentaram um aumento de aproximadamente $4 \%$ de produtividade em relação ao ano anterior, demonstrando assim uma tendência de aumento no mercado, principalmente na região Sul do país, onde estão concentradas as indústrias de painéis de madeira e polos moveleiros (IBÁ, 2020).

Reconhecendo assim a potencialidade da modificação térmica, e dado que as características tecnológicas da madeira geram parâmetros adequados para avaliar a sua qualidade, este estudo teve como objetivo analisar o efeito da modificação térmica sobre as propriedades físicas da madeira de Pinus caribaea var. hondurensis Barrett \& Golfari através dos ensaios de densidade aparente, umidade, perda de massa, taxa de absorção de água, testes de contração e inchamento volumétrico, e coeficiente de anisotropia.

\section{Material e métodos}

\section{Obtenção do material e confecção dos corpos de prova}

O estudo foi conduzido no Laboratório de Tecnologia de Produtos Florestais da Universidade Estadual do Sudoeste da Bahia (UESB), campus de Vitória da Conquista, BA. Foram utilizadas três árvores de Pinus caribaea var. hondurensis Barrett \& Golfari com idades de 14 anos, cultivadas no campo experimental da UESB, com espaçamento de $3 \times 3 \mathrm{~m}$, nas coordenadas geográficas de $14^{\circ} 53^{\prime} 12^{\prime \prime}$ e e $40^{\circ} 48^{\prime} 7^{\prime \prime}$ 'W. A região de Vitória da Conquista tem altitude relativa de aproximadamente $900 \mathrm{~m}$, clima caracterizado como tropical de altitude (Cwb), segundo a classificação de Köppen; temperatura média anual de $21^{\circ} \mathrm{C}$, com precipitação pluviométrica irregular, variando entre 700 e $1.100 \mathrm{~mm}$ anuais (Novaes et al., 2007).

As árvores selecionadas possuíam alturas e DAP (diâmetro à altura do peito) semelhantes, com médias de $12,6 \mathrm{~m}$ e 17,80 cm, respectivamente. Após a derrubada, foram retirados discos de dez centímetros de espessura a $0 \%$ (iniciando a $10 \mathrm{~cm}$ de altura do solo), $25 \%$ e $50 \%$ da altura comercial e na altura do DAP. Utilizaram-se discos até $50 \%$ da altura comercial por se tratar de árvores de pequeno diâmetro. Os corpos de provas foram divididos em três lotes iguais, com 36 amostras para cada, nas dimensões de 30 x 30 x $50 \mathrm{~mm}$ (espessura x largura x comprimento), conforme a NBR 7190 (ABNT, 1997).

\section{Processo de modificação térmica}

Os corpos de prova, com umidade inicial de $12 \%$, foram modificados termicamente a $160^{\circ} \mathrm{C}$ e $200^{\circ} \mathrm{C}$ em uma estufa com circulação e renovação de ar. Um dos lotes não passou pelo processo, sendo assim utilizado para o controle.

O programa utilizado no processo de modificação térmica (Tabela 1) foi desenvolvido com base na literatura (Modes et al., 2013; Poubel et al., 2013; Nunes et al., 2016; Santos et al., 2016). Após o processo, as amostras 
Efeito da modificação térmica nas propriedades físicas da madeira de Pinus Caribaea var. Hondurensis Barrett \& Golfari

foram aclimatizadas e, posteriormente, determinadas

suas propriedades físicas.

Tabela 1 - Programa utilizado no processo de modificação térmica das madeiras de Pinus caribaea var. hondurensis Barrett \& Golfari.

\begin{tabular}{|c|c|c|}
\hline Etapas & Ciclos de temperatura & Tempo \\
\hline I & Aquecimento da temperatura inicial de $30^{\circ} \mathrm{C}$ até $100^{\circ} \mathrm{C}$ & 30 minutos \\
\hline II & Manutenção de $100^{\circ} \mathrm{C}$ & 120 minutos \\
\hline III & Aquecimento até a temperatura final $\left(160^{\circ} \mathrm{C}\right.$ e $\left.200^{\circ} \mathrm{C}\right)$ & $\begin{array}{c}30 \text { a } 60 \text { minutos, a depender da } \\
\text { temperatura final }\end{array}$ \\
\hline IV & Manutenção da temperatura final $\left(160^{\circ} \mathrm{C}\right.$ e $\left.200^{\circ} \mathrm{C}\right)$ & 120 minutos \\
\hline $\mathrm{V}$ & Resfriamento & 120 minutos \\
\hline
\end{tabular}

\section{Determinação das propriedades físicas}

Foram utilizadas 12 amostras de cada tratamento térmico e 12 de controle para os ensaios de umidade, densidade e perda de massa; 12 para a taxa de absorção de água; e 12 para os ensaios de contração e inchamento volumétrico, e coeficiente de anisotropia.

O teor de umidade foi medido de acordo com a NBR 14929 (ABNT, 2003), pela diferença de massa das amostras antes e depois da secagem em estufa a $103 \pm 2^{\circ} \mathrm{C}$ até massa constante. $\mathrm{O}$ cálculo do teor de umidade da madeira foi realizado pela equação 1 .

$$
\left.T . U=\frac{(M u-M s)}{M s} * 100 \quad \text { (Eq. } 1\right)
$$

Em que: T.U. = teor de umidade (\%); $\mathrm{Mu}=$ massa úmida (g); e Ms $=$ massa seca $(\mathrm{g})$.

A densidade aparente anidra foi determinada de acordo com a recomendação e adaptação da NBR 7190 (ABNT, 1997), em que a massa absolutamente seca da madeira foi medida em uma balança de precisão e o volume foi medido por meio das dimensões da amostra com o auxílio de um paquímetro digital. $\mathrm{O}$ cálculo foi realizado pela equação 2 .

$$
D a=\frac{M}{V} \quad \text { (Eq. 2) }
$$

Em que: Da $=$ densidade aparente anidra $\left(\mathrm{g} / \mathrm{cm}^{3}\right) ; \mathrm{M}=$ massa seca (g); e V = volume da amostra $\left(\mathrm{cm}^{3}\right)$.

A perda de massa foi determinada apenas para as amostra tratadas termicamente, pela relação entre a diferença da massa seca antes e após a modificação térmica, sendo calculada pela equação 3 .

$$
P . M .=\frac{(M n t-M t)}{M n t} * 100 \quad \text { (Eq. 3) }
$$

Em que: P.M. = perda de massa (\%); Mnt = massa seca antes do tratamento (g); e Mt = massa seca após o tratamento $(\mathrm{g})$.

Para a taxa de absorção de água, as amostras foram imersas em água por 24 horas e, posteriormente, passaram pelo processo de secagem em estufa a $103 \pm 2^{\circ} \mathrm{C}$ até peso constante. Sendo assim calculada pela equação 4.

$$
T . A .=\frac{(M 1-M 2)}{M 2} * 100 \text { (Eq. 4) }
$$

Em que: T.A. = taxa de absorção de água (\%); M1 = massa úmida (g); e M2 = massa seca $(\mathrm{g})$.

O ensaio de estabilidade dimensional foi realizado de acordo com a NBR 15261 (ABNT, 2005), onde as amostras foram imersas em água até saturação completa e as dimensões foram mensuradas com um paquímetro digital nos três sentidos estruturais (longitudinal, tangencial e radial). As mesmas foram conduzidas a estufa a $103 \pm 2^{\circ} \mathrm{C}$ durante 24 horas e as dimensões novamente mensuradas. Assim, foram determinadas a contração volumétrica $(\beta \mathrm{v})$, contração de inchamento volumétrico ( $\alpha$ v) e o coeficiente de anisotropia (C.A.) pelas equações 5,6 e 7 , respectivamente.

$$
\begin{gathered}
\beta v=\frac{(V u-V 0)}{V u} * 100 \quad \text { (Eq. 5) } \\
\alpha v=\frac{(V u-V 0)}{V 0} * 100 \quad \text { (Eq. 6) } \\
\text { C.A. }=\frac{\beta t}{\beta r} \quad \text { (Eq. 7) }
\end{gathered}
$$

Em que: $\beta \mathrm{v}=$ contração volumétrica (\%); $\mathrm{Vu}=$ volume úmido $(\mathrm{mm})$; $\mathrm{V} 0=$ volume seco $(\mathrm{mm}) ; \alpha \mathrm{v}=$ inchamento volumétrico (\%); C.A. = coeficiente de anisotropia; $\beta(\mathrm{t}, \mathrm{r})=$ coeficiente de contração linear.

\section{Análise estatística}

Os resultados foram analisados em função do efeito da modificação térmica nas propriedades físicas da madeira. Foram avaliados por meio da análise de variância e do teste de Tukey, ambos a 5\% de probabilidade, utilizando-se o programa Excel ${ }^{\circledR}$.

\section{Resultados e discussão}

Os valores médios para a umidade e taxa de absorção de água da madeira modificada termicamente de Pinus caribaea var. hondurensis Barrett \& Golfari apresentaram resultados menores que àqueles obtidos 
Santos, V. B. e Silva, G. C.

para o controle, contudo, não houve diferença estatística entre as temperaturas de tratamento. Em relação à perda de massa, as madeiras modificadas apresentaram resultados significativos, sendo que, as maiores médias foram encontradas para a maior temperatura $\left(200^{\circ} \mathrm{C}\right)$.
Entretanto, mesmo a amostras modificadas termicamente apresentarem um menor valor para a densidade aparente, não houve diferença significativa entre os tratamentos estudados (Tabela 2).

Tabela 2 - Médias e coeficiente de variação (\%) dos parâmetros avaliados da madeira de Pinus caribaea var. hondurensis Barrett \& Golfari.

\begin{tabular}{lcccc}
\hline Tratamentos & $\begin{array}{c}\text { T.U. } \\
\mathbf{( \% )}\end{array}$ & $\begin{array}{c}\text { Da } \\
\left(\mathbf{g} / \mathbf{c m}^{3}\right)\end{array}$ & $\begin{array}{c}\text { T.A. } \\
\mathbf{( \% )}\end{array}$ & $\begin{array}{c}\text { P. M. } \\
\mathbf{( \% )}\end{array}$ \\
\hline \multirow{2}{*}{ Controle } & $12,0 \mathrm{a}$ & $0,42 \mathrm{a}$ & $80,06 \mathrm{a}$ & - \\
& $(3,55)$ & $(6,73)$ & $(10,03)$ & - \\
$160^{\circ} \mathrm{C}$ & $7,88 \mathrm{~b}$ & $0,40 \mathrm{a}$ & $74,97 \mathrm{~b}$ & $5,55 \mathrm{a}$ \\
& $(1,30)$ & $(8,46)$ & $(3,06)$ & $(5,09)$ \\
$200^{\circ} \mathrm{C}$ & $6,42 \mathrm{~b}$ & $0,40 \mathrm{a}$ & $70,95 \mathrm{~b}$ & $8,20 \mathrm{~b}$ \\
& $(6,32)$ & $(5,91)$ & $(3,04)$ & $(4,99)$ \\
\hline
\end{tabular}

Em que: T.U = teor de umidade; $\mathrm{Da}=$ densidade aparente; T.A. = taxa de absorção de água; P.M = perda de massa. Médias seguidas por uma mesma letra em uma mesma coluna não diferem estatisticamente entre si pelo Teste de Tukey à $5 \%$ de significância.

A redução no teor de umidade da madeira de $P$. caribaea var. hondurensis está diretamente relacionada com as alterações químicas que a modificação térmica promove nos constituintes da parede celular, principalmente dos grupos hidroxilas, como por exemplo, a celulose e hemicelulose, tornando assim a madeira menos higroscópica (Kocaefe et al., 2008; Lengowski et al., 2018). Neste sentido, a taxa de absorção de água das madeiras tratadas neste trabalho demonstra a eficiência e importância da modificação térmica na redução da higroscopicidade, visto que, seus valores foram estatisticamente menores que as do controle, havendo assim, uma tendência de decréscimo conforme o aumento da temperatura.

A perda de água livre e higroscópica presente nas paredes celulares da madeira, juntamente com a decomposição dos seus constituintes químicos, também influenciaram na perda de massa das amostras. Além de que, madeiras de menor densidade, como as do gênero Pinus, espera-se maior redução percentual de massa quando submetidas a tratamentos térmicos, pois, as mesmas têm menos massa por unidade de volume (Hill, 2006.; Lobato et al., 2020).

Assim, resultados semelhantes aos dos parâmetros estudados foram encontrados por Bellon (2013), que ao trabalhar com madeiras de Pinus taeda tratadas a $160^{\circ} \mathrm{C}$ obteve umidade final de $7,60 \%$ e densidade de $0,41 \mathrm{~g} / \mathrm{cm}^{3}$; e por Moura et al. (2011) e Schulz et al. (2020) trabalhando a $200^{\circ} \mathrm{C}$ com Pinus caribaea var. hondurensis e Pinus elliottii, obtiveram 7,51\% e 7,38\%, respectivamente, de redução de massa.

Os valores das densidade aparente não terem sido significativos (Tabela 2), manifestou a mesma tendência dos estudos de Conte et al. (2014), Juizo et al. (2018), Lobato et al. (2020) e Schulz et al. (2020), onde analisaram diferentes tratamentos de modificação térmica para as madeiras de Pinus elliottii, Eucalyptus sp., Pouteria caimito e $P$. elliottii, respectivamente, e não apresentaram diferença para esse parâmetro. Isto indica que, durante o processo de modificação térmica, o aumento de temperatura não provocou nas amostras uma diminuição de massa proporcional à diminuição de volume, apontando assim, que a densidade aparente não se apresentou como uma boa propriedade para avaliar a qualidade do tratamento térmico (Calonego et al., 2012).

Em relação à estabilidade dimensional, o processo de modificação térmica reduziu significativamente a contração volumétrica e o inchamento volumétrico da madeira de Pinus caribaea var. hondurensis, proporcionando-a assim uma menor instabilidade dimensional, porém, não houve diferença estatística entre as temperaturas de tratamento. Já para o coeficiente de anisotropia, o procedimento térmico não influenciou no resultado final (Tabela 3).

Os fenômenos de inchamento e contração que ocorrem na madeira devido a entrada e saída de água de adesão podem causar defeitos nas peças, como rachaduras e fendilhamentos. Neste sentido, reduzir a instabilidade dimensional da madeira por meio de tratamentos térmicos, como no presente estudo, melhora a sua qualidade para determinados setores, como construção civil, movelaria, decoração entre outros (He et al., 2019).

A eficiência do aumento da estabilidade dimensional nas amostras de $P$. caribaea var. hondurensis pode ser explicada pela degradação das hemiceluloses, que é o componente estrutural mais hidrofílico, e, consequentemente redução da higroscopicidade. Já que, a menor retratibilidade ocorre pela redução dos sítios de sorção, sobretudo das hidroxilas, que se envolvem com as moléculas de água durante as trocas de umidade com o ambiente externo (Jämsä e Viitaniemi, 2001; Kocaefe 
Efeito da modificação térmica nas propriedades físicas da madeira de Pinus Caribaea var. Hondurensis Barrett \& Golfari

et al., 2015). Valores similares foram apresentados por Conte et al. (2014), 8,25\% de contração volumétrica, com madeiras de Pinus elliottii var. elliottii tratadas a $210^{\circ} \mathrm{C}$; e por Poubel et al. (2013), 8,16\% de inchamento volumétrica, com madeiras de Pinus caribaea tratadas a $200^{\circ} \mathrm{C}$.

Tabela 3 - Médias e coeficiente de variação (\%) da contração volumétrica, inchamento volumétrico e coeficiente de anisotropia dos tratamentos de Pinus caribaea var. hondurensis Barrett \& Golfari.

\begin{tabular}{lccc}
\hline Tratamentos & $\beta \mathrm{v}(\%)$ & $\alpha \mathrm{v}(\%)$ & C.A. \\
\hline \multirow{2}{*}{ Controle } & $13,52 \mathrm{a}$ & $15,66 \mathrm{a}$ & $0,89 \mathrm{a}$ \\
& $(9,62)$ & $(11,21)$ & $(9,63)$ \\
$160^{\circ} \mathrm{C}$ & $9,32 \mathrm{~b}$ & $10,22 \mathrm{~b}$ & $0,86 \mathrm{a}$ \\
& $(18,37)$ & $(19,03)$ & $(16,57)$ \\
$200^{\circ} \mathrm{C}$ & $8,20 \mathrm{~b}$ & $8,98 \mathrm{~b}$ & $0,86 \mathrm{a}$ \\
& $(18,16)$ & $(19,23)$ & $(18,01)$ \\
\hline
\end{tabular}

Em que: $\beta v$ = contração volumétrica; $\alpha \mathrm{v}=$ inchamento volumétrico; C.A. = coeficiente de anisotropia. Médias seguidas por uma mesma letra em uma mesma coluna não diferem estatisticamente entre si pelo Teste de Tukey à $5 \%$ de significância.

Mesmo não apresentando diferença significativa entre os tratamentos estudados, os valores do coeficiente de anisotropia demostraram que a espécie de $P$. caribaea var. hondurensis apresenta uma madeira de boa qualidade (Tabela 3), visto que, quanto mais próximo de 1 melhor é a qualidade da madeira, já que um coeficiente de anisotropia igual a 1 representa uma alteração igual de dimensões nos sentidos radial e tangencial, situação considerada ideal e que não provocaria formação de tensões internas (Durlo e Marchiori, 1992). Resultados para o coeficiente de anisotropia apresentados por Bellon (2013) e Candaten et al. (2020), em madeiras tratadas de Pinus taeda e Handroanthus chrysotrichus, respecti- vamente, indicaram que a modificação térmica também não alterou esta propriedade física.

Quanto ao aspecto visual das amostras estudadas, verificou-se que a modificação térmica também influenciou, visualmente, na coloração da madeira, principalmente àquelas tratadas a $200^{\circ} \mathrm{C}$ (Figura 1). Quirino (2003), explica que espécies de baixa densidade, como as do gênero de Pinus, adquirem uma maior alteração na cor original das peças após a modificação térmica. Isto ocorre pela combinação de vários fatores, como a formação de produtos oxidativos e produtos da degradação das hemiceluloses e lignina, os quais migram às superfícies da madeira (Esteves et al., 2008).

Figura 1 - Corpos de prova da madeira de Pinus caribaea var. hondurensis Barrett \& Golfari.

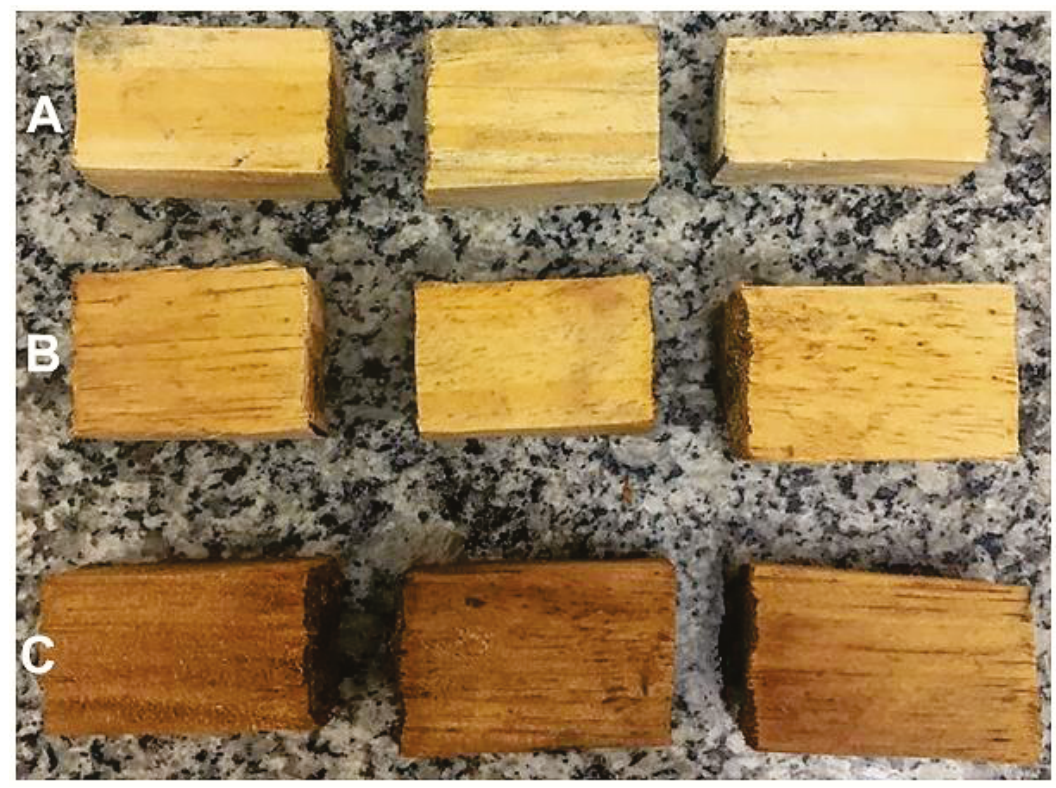

A: Controle; B: modificadas termicamente a $160^{\circ} \mathrm{C}$; C: modificadas termicamente a $200^{\circ} \mathrm{C}$.

Lengowski et al. (2018), Mendoza et al. (2020) e Schulz et al. (2020), também constataram a alteração da cor das peças de madeira após os diferentes processos de modificação térmica, sendo a maior variação da cor observada no tratamento de maior temperatura final. Neste sentido, a modificação térmica demonstra ser um processo vantajoso, pois, além de ser eficiente na melhoria das propriedades físicas da madeira, ainda agrega valor 
as suas peças, já que, madeiras com coloração escura tendem a apresentar maior valor comercial.

\section{Conclusão}

O processo de modificação térmica influenciou nas propriedades avaliadas da madeira de Pinus caribaea var. hondurensis Barrett \& Golfari. Houve alteração das características físicas, que, em função da redução da umidade de equilíbrio, associada a uma maior perda de massa, tornou a madeira menos higroscópica e com maior estabilidade dimensional, sendo estes valores mais evidentes na exposição a maior temperatura. Além de ter influenciado visualmente na coloração da madeira em relação a sua aparência natural, agregando assim, valor nas peças e as potencializando para diversos setores, como para fins estruturais, aplicações exteriores e setor moveleiro.

\section{Agradecimentos}

Os autores agradecem à Universidade Estadual do Sudoeste da Bahia (UESB) e ao Programa de Iniciação Científica (PIC/UESB).

\section{Referências}

Associação Brasileira de Normas Técnicas (ABNT). 1997. NBR 7190: Projeto de Estruturas de Madeira. Rio de Janeiro.

Associação Brasileira de Normas Técnicas (ABNT). 2003. NBR 14929: Determinação do teor de umidade da madeira. Rio de Janeiro.

Associação Brasileira de Normas Técnicas (ABNT). 2005. NBR 15261: Determinação da variação dimensional da madeira. Rio de Janeiro.

Batista, D. C. 2019. Retificação térmica, termorretificação, tratamento térmico, tratamento com calor ou modificação térmica? Ciência Florestal, 29:463-480. Doi: https://doi.org/10.5902/1980509822577.

Bellon, K. R. R. 2013. Modificação térmica da madeira de três espécies de florestas plantadas pelo processo VAP HolzSysteme®. Curitiba: Universidade Federal do Paraná, 101f. Dissertação Mestrado. Disponível em: https://acervodigital.ufpr.br/handle/1884/37706.

Calonego F. W.; Severo, E. T. D.; Ballarin, A. W. 2012. Physical and mechanical properties of thermally modified wood from E. grandis. European Journal of Wood and Wood Products, 70:453-460. Doi: https://doi.org/10.1007/s00107-011-0568-5.

Candaten, L.; Costa, H. W. D.; Trevisan, R.; Eloy, E.; Kulcynski, S. M. 2020. Physical-mechanical properties and biological resistance of thermally modified juvenile Handroanthus chrysotrichus wood. Revista de Ciências Agrárias, 63:1-8. Disponível em: https://periodicos.ufra. edu.br/index.php/ajaes/article/view/3258.

Conte, B.; Missio A. L.; Pertuzzatti, A.; Cademortori, P. H. G.; Gatto, D. A. 2014. Propriedades físicas e colorimétricas da madeira termorretificada de Pinus elliottii var. elliottii. Scientia Forestalis, 42: 555-563. Disponível em: https://www.ipef.br/publicacoes/scientia/nr104/cap09.pdf.

Durlo, M. A.; Marchiori, J. N. C. Tecnologia da madeira: retratibilidade. Santa Maria: CEPEF/FATEC, 1992. 33p. (Série Técnica, 10).

Esteves, B.; Velez, M. A.; Domingos, I.; Pereira, H. 2008. Heat induced colour changes of pine (Pinus pinaster) and eucalyptus (Eucalyptus globulus) wood. Wood Science and Technology 42: 369-384. Doi: https://doi.org/10.1007/s00226-007-0157-2.

Esteves, B. M.; Pereira, H. M. 2009. Wood modification by heat treatment: a review. BioResources, 4: 370-404. Disponível em: https://ojs.cnr.ncsu. edu/index.php/BioRes/article/view/BioRes_04_1_\%23\%23\%23\%23 Esteves_P_Wood_Mod_Heat_Treatment.

He, Z.; Qu, L.; Wang, Z.; Qian, J.; Yi, Y. 2019. Effects of zinc chloridesilicone oil treatment on wood dimensional stability, chemical components, thermal decomposition and its mechanism. Scientific Reports, 9:e1601. Doi: https://doi.org/10.1038/s41598-018-38317-5.

Hill, C. A. S. 2006. Wood Modification: Chemical, Thermal and Other Processes. Chichester: John Wiley \& Sons.
Indústria Brasileira de Árvores, IBÁ: Relatório 2020 - Ano base 2019. São Paulo, SP, 2020. Disponível em: https://iba.org/datafiles/publicacoes/ relatorios/relatorio-iba-2020.pdf.

International Thermowood Association. 2020. Thermowood production statistics 2019. Disponível em: https://asiakas.kotisivukone.com/files/ en.thermowood.palvelee.fi/uutiset/Productionstatistics2019.pdf.

Jämsä, S.; Viitaniemi, P. 2001. Heat treatment of wood - Better durability without chemicals. In: Proceedings of special seminar held in Antibes, France.

Juizo, C. G. F.; Zen, L. R.; Klitzke, W.; França, M. C.; Cremonez, V. G.; Klitzke, R. J. 2018. Propriedades tecnológicas da madeira de eucalipto submetida ao tratamento térmico. Nativa, Sinop, 6: 537-542. Doi: http://dx.doi.org/10.31413/nativa.v6i5.5666.

Kamdem, D. P.; Pizzi, A.; Jermannaud, A. 2002. Durability of heattreated Wood. Holz als Roh-und Werkstoff. Berlin: Springer-Verlag. p.1-6. Doi: https://doi.org/10.1007/s00107-001-0261-1.

Kocaefe, D.; Poncsák, S.; Doré, G.; Younsi, R. 2008. Effect of heat treatment on the wettability of white ash and softmaple by water. Holz als Roh-und Werkstoff, 66: 355-361. Doi: https://doi.org/10.1007/ s00107-008-0233-9.

Kocaefe, D.; Huang, X.; Kocaefe, Y. 2015. Dimensional Stabilization of Wood. Current Forestry Reports, 1 : 151 -161. Doi: https://doi. org/10.1007/s40725-015-0017-5.

Lengowski, E. C.; Muñiz, G. I. B.; Klock, U.; Nisgoski, S. 2018. Potential use of nir and visible spectroscopy to analyze chemical properties of thermally treated wood. Maderas, Ciencia y Tecnología, Concepción, 20:627-640. Doi: http://dx.doi.org/10.4067/S0718221X2018005041001.

Lobato, C. C. S.; Do Vale, I.; Souza, L. M.; Abreu, J. L. L.; Ferreira, G. C.; Bufalino, L. 2020. Densidade básica e estabilidade dimensional de madeiras tropicais modificadas por tratamentos térmicos. Nativa, Sinop, 8: 708-714. Doi: https://doi.org/10.31413/nativa.v8i5.10630.

Mendoza, Z. M. S. H.; Borges, P. H. M.; Morais, P. H. M. 2020. Modificação térmica em madeiras tropicais. Enciclopédia Biosfera, Centro Científico Conhecer - Goiânia, 17:382. Doi: https://doi.org/10.18677/ EnciBio_2020B34.

Modes, K. S.; Santini, E. J.; VIVIAN, M. A. 2013. Hygroscopicity of wood from Eucalyptus grandis and Pinus taeda subjected to thermal treatment. Cerne, Lavras, 19: 19-25. Doi: https://doi.org/10.1590/ S0104-77602013000100003.

Modes, K. S.; Santini, E. J.; Vivian, M. A.; Garlet, A. 2017. Influência da termorretificação na resistência a degradação biológica das madeiras de Pinus taeda e Eucalyptus grandis. Ciência Florestal, 27: 993-1002. Doi: https://doi.org/10.5902/1980509828672. 
Efeito da modificação térmica nas propriedades físicas da madeira de Pinus Caribaea var. Hondurensis Barrett \& Golfari

Moura, L. F.; Brito, J. O.; Bortoletto Júnior,G. 2011. Efeitos da termorretificação na perda de massa e propriedades mecânicas de Eucalyptus grandis e Pinus caribaea var. hondurensis. Floresta, 42: 305 - 314. Doi: http://dx.doi.org/10.5380/rf.v42i2.17635.

Novaes, A. B.; Longuinhos, M. A. A.; Rodrigues, J.; Santos, I. F.; Gusmão, J. C. 2007. Caracterização e demanda florestal da Região Sudoeste da Bahia. In: Santos, A. F.; 422 Novaes, A.B.; Santos, I.F.; Longuinhos, M.A.A. (Org.). Memórias do II Simpósio sobre Reflorestamento na Região Sudoeste da Bahia. 1⿳⺈ ed. Colombo: Embrapa Florestas, 1: 25-43.

Nunes, C. S.; Nascimento, A. M.; Garcia, R. A.; Lelis, R. C. C. 2016. Qualidade de adesão das madeiras de Corymbia citriodora e Eucalyptus pellita modificadas termicamente. Scientia Forestalis, 44: 41-56. 2016. Doi: http://dx.doi.org/10.18671/scifor.v44n109.04.
Poubel, D. S.; Garcia, R. A.; Santos, W. A.; Oliveira, G. L.; Abreu, H. S. 2013. Efeito da termorredificação nas propriedades físicas e químicas da madeira de Pinus caribae. Cerne, 19: 391-398. Doi: http://dx.doi. org/10.1590/S0104-77602013000300005.

Quirino, W. F. 2003. Utilização energética de resíduos vegetais. Brasília, DF: IBAMA/LPF. Disponível em: http://www.mundoflorestal.com.br/ arquivos/aproveitamento.pdf.

Santos, P. V.; Silva, M. S.; Dias Junior, A. F.; Carvalho,A. M.; Nascimento, A. M.; 2016. Qualidade tecnológica da madeira de Corymbia citriodora (Hook.) K.D. Hill \& L.A.S. Johnson submetida ao processo de termorretificação. Scientia Forestalis, 44: 343-350. Doi: https://doi. org//10.18671/scifor.v44n110.07.

Schulz, H. R.; Acosta, A. P.; Barbosa, K. T.; Gallio, E.; Beltrame, R.; Gatto, D. A. 2020. Efeito do tratamento térmico da madeira de Pinus elliottii nos parâmetros físico-mecânicos e colorimétricos. Biofix Scientific Journal, 5: 86-93. Doi: https://doi.org//10.5380/biofix.v5i1.68111. 